\title{
Canestriniidae (Acari, Astigmata) of Poland, with key to species of the genus Photia Oudemans, 1904
}

\author{
Ryszard HAITLINGER \\ Institute of Biology, Department of Invertebrates Systematics and Ecology, Wroctaw University of Environmental and \\ Life Sciences, Kożuchowska 5B, 51631 Wroctaw; \\ ryszard.haitlinger@up.wroc.pl
}

\begin{abstract}
In Poland 14 species of canestriniid mites were found. The total number of collected mites were 1693. Photia hejniana and $P$. chrysocarabi are most numerous species in the country. Only $P$. hejniana was stated in the whole country. Very rare species are Percanestrinia blaptis, Dicanestrinia knobi, D. huberti, Coleopterophagus albini and Pseudocanestrinia mahunkai. All these species are associated with rare host species in Poland. Key to the Photia species of the world is provided.
\end{abstract}

Key words: Acari, Canestriniidae, faunistic list, Poland, Photia

\section{INTRODUCTION}

The family Canestriniidae contains about 95 genera and 305 species. In Poland the family Canestriniidae is hitherto poorly known. First record of canestriniid mites was given by Haitlinger (1988a, b). Then stated in Poland 3 species: Canestrinia dorcicola Berlese, 1881, Dicanestrinia knobi Samšiňák, 1971 and Coleopterophagus megnini (Berlese, 1881). Later 11 further species were found; among them four species were new for sciences: Photia bardoica Haitlinger, 1988, P. hermengildae Haitlinger, 1988, P. adolfinae Haitlinger, 1994 and Coleopterophagus albini Haitlinger, 1990 (Haitlinger, 1988c, d, 1990. 1994, 2002, 2004). P. polymorpha Samšiňák, 1971 earlier mentioned from Poland was mistakenly determined; these specimens belong to P. adolfinae. P. polymorpha in Poland is absent. All canenistriid mites occurring in Poland are associated with various species of Carabidae, Scarabaeidae, Tenebrionidae and Lucanidae (Insecta: Coleoptera). Now, 14 species of canenistriid mites belonging to 7 genera are known from Poland. In this paper all known localities in Poland are mentioned and new localities are given. In the absence of any key to the world canestriniid species, and that the other keys are restricted only to small regions, the key to the Photia species of the world is provided.

\section{MATERIAL AND METHODS}

In this paper besides earlier published data are given new information on canestriniid mites living in Poland. The analyzed material of hosts and mites was collected in various localities over the whole country since 1983 till 2009. These beetles (Coleoptera) were taken from the soil, under of stones, under bark of trees and traps and later mites were taken from their body. Mites were preserved in $70 \%$ ethanol. The following species of Coleoptera were investigated: Carabus coriaceus Linnaeus, 1758, C. violaceus Fabricius, 1787, C. scheidleri Panzer, 1799, C. linnaei Panzer, 1812, C. glabratus Paykull, 1790, C. nemoralis O. F. Müller, 1764, C. auronitens Fabricius, 1792, C. variolosus Fabricius, 1787 (Carabidae), Dorcus paralellopipedus (Linnaeus, 1758) (Lucanidae), Blaps lethifera Marsham, 1802 (Tenebrionidae), Protaetia (Potosia) 
metallica (Herbst, 1782) and P. (Cetonischema) aeruginosa (Drury, 1770) (Scarabaeidae).The total number of collected mites were 1693 (104 C. sellnicki, 1 P. mahunkai, 43 P. bourgognei, 348 P. chrysocarabi, 708 P. hejniana, 171 P. hermengildae, 42 P. adolfinae, 276 P. bardoica). The idiosomal chaetotaxy nomenclature used in key is based on Griffiths et al. (1990).

\section{RESULTS}

Canestrinia Berlese, 1881

\section{Canestrinia dorcicola Berlese, 1881}

Localities: Krzywa Góra n. Pokój (Opolskie prov.), Nowogród Bobrzański n. Zielona Góra (Lubuskie prov.), Sẹdziszów Małopolski (Podkarpackie prov.), Kępice n. Slupsk (Pomorskie prov.) (Haitlinger, 1988a, 2004).

Host: Dorcus paralellopipedus (Linnaeus, 1758).

Distribution: Italy, Poland, Ukraine (Haitlinger,1988a, Khaustov \& Eidelberg, 2001, Trach, 2006).

Pseudocanestrinia Khaustov \& Eidelberg, 2001

\section{Pseudocanestrinia mahunkai (Samšiňák, 1971)}

Localities: Wroclaw-Osobowice, Olawa (Dolnośląskie prov.) (Haitlinger, 1988d).

New locality: 1q, 19.09.1991, Porażyn n. Opalenica (Wielkopolskie prov.)

Host: Carabus coriaceus.

Distribution: Hungary. Poland (Samšiňák, 1971, Haitlinger, 1988d).

Dicanestrinia Berlese, 1911

\section{Dicanestrinia knobi Samšiňák, 1971}

Localitiy: Pieniny (Małopolskie prov.) (Haitlinger, 1988b).

Host: Carabus variolosus Fabricius, 1787.

Distribution: Czech Republic, Yugoslavia, Italy, Poland, Ukraine (Samšiňák, 1971, Haitlinger, 1988a, Trach, 2006).

\section{Dicanestrinia huberti Haitlinger, 1994}

Locality: Bystre n. Baligród (Podkarpackie prov.) (Haitlinger, 1994).

Host: Carabus variolosus.

Distribution: Byelorussia, Poland (Haitlinger, 1994, Khaustov \& Eidelberg, 2001).

Coleopterophagus Berlese, 1882

\section{Coleopterophagus megnini (Berlese, 1881)}

Localities: (from Cetonia aurata) Kamionka n. Kolbuszowa, Huwniki n. Przemyśl, (from Protaetia (P.) metallica) Kalnica n. Wetlina, Raniżów n. Kolbuszowa, Łowisko n. Leżajsk, , Berezka n. Lesko, Huta Różaniecka n. Narol, Żohatyń n. Bircza (Podkarpackie prov.), Ustrzyk n. Ochotnica Dolna (Malopolskie prov.), Bobolice n. Myszków, Podzamcze n. Kroczyce (Śląskie prov.), Stare Łysogórki n. Moryń, Wicie n. Jaroslawiec (Zachodniopomorskie prov.), Górecko Stare n. Zwierzyniec (Lubelskie prov.), Wola Klucka n. Strawczyn (Świętokrzyskie prov.), Skłoby n. Szydłowiec (Mazowieckie prrov.), Dobrylas n. Łomża (Podlaskie prov.), 
Męcikal n. Brusy, Struga n. Męcikal, Leśno n. Brusy (Pomorskie prov.), Wroclaw-Swojczyce (Dolnośląskie prov.) (Haitlinger, 1988b, 2002).

Hosts: Protaetia (Potosia) metallica (Herbst, 1782), P. (P.) cuprea (Fabricius, 1775), P. (Eupotosia) affinis (Andresch, 1797), P. (Potosia) fieberi (Kraatz, 1880), P. (Potosia) cuprina (Motschulsky, 1849), Cetonia aurata (Linnaeus, 1761). C. aurata is accidental host..

Distribution: Czech Republic, Croatia, England, Hungary, Holland, Italy, Poland, Turkey, Ukraine, Yugoslavia (Haitlinger, 1990, Khauatov \& Eidelberg, 2001, Trach, 2006, Trach \& Khaustov, 2011).

\section{Coleopterophagus albini Haitlinger, 1990}

Localities: Zaborze n. Połczyn Zdrój (Zachodniopomorskie prov.), Balamątek n. Rowy (Pomorskie prov.) (Haitlinger, 1990, 2002).

Hosts: Protaetia (Cetonischema) aeruginosa (Drury, 1770), P. (Liocola) marmorata (Fabricius, 1792).

Distribution: Austria, Czech Republic, Germany, Poland, Romania, Ukraine (Haitlinger, 1990, Trach \& Khaustov, 2011).

Procericola Cooreman, 1950

\section{Procericola bourgognei (Oudemans, 1923)}

Localities: Zabór Wielki, Olawa, Wrocław (Dolnoślaskie prov.), Rogalice n. Brzeg, Lubsza (Opolskie prov.), Rogacz n. Klimontów (Świętokrzyskie prov.), Policzno n. Puck (Pomorskie prov.) (Haitlinger, 1988d).

New localities: $19,3 \hat{\jmath}, 19.09 .1991$, Porażyn n.. Opalenica (Wielkopolskie prov.) (C.

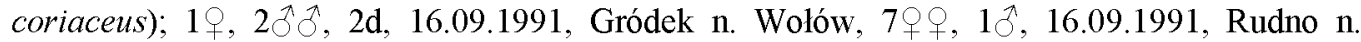
Wolów (Dolnośląskie prov.); 1§, 3d, 25.05.1988, Przyłubie n. Solec Kujawski (KujawskoPomorskic prov.); 3ㅇ, $2 \AA$ \%, 18.08.1990, Kluczewo n. Czaplinek (Zachodniopomorskie prov.); 2우, 2ええ, 22.09.1993, Niżankowice n. Działoszyn (Lódzkie prov.); 5우, 4えð, 5.08.1996, Kamionek Wielki n. Tolkmicko (Warmińsko-Mazurskie prov.); 2q9, 2ठึฎ, 5.10.2009, Ćmielów (Świętokrzyskie prov.)

Host: Carabus coriaceus.

Distribution: Caucasus, Czech Republic, Moldova, Poland, Ukraine (Samšinák, 1971, Haitlinger, 1988d, Khaustov \& Eidelberg, 2001, Trach, 2006).

Percanestrinia Berlese, 1911

\section{Percanestrinia blaptis (G. Canestrini \& Berlese, 1880)}

Locality: Ukta n. Ruciane (Warmińsko-Mazurzskie prov.) (Haitlinger, 1992)

Hosts: Blaps lethifera Marsham, 1802, B. punctata, B. occulta Seidlitz, 1833, B. gibbosa, Sphodrus leucophthalmus (Linnaeus, 1758).

Distribution: Bulgaria, Georgia, Germany, Hungary, Italy, Poland, Ukraine (Samšiňák, 1965, Haitlinger, 1992, Khaustov \& Eidelberg, 2001).

\section{Percanestrinia sellnicki (Samšiňák, 1971)}

Localities: Zabór Wielki, Sulistrowiczki n. Sobótka, Wroclaw, Olawa (Dolnoślaskie prov.), Moszna, Rogalice n. Brzeg, Kamień Śląski (Opolskie prov.), Podgaje n. Jastrowie (Wielkopolskie prov.), Bieszczady (Podkarpackie prov.) (Haitlinger, 1988d).

New localities:. 9qq, $3 \hat{\jmath}, 9 \mathrm{~d}, 19.09 .1991$, Porażyn n. Opalenica ( from C. coriaceus) (Wielkopolskie prov.), 2qq, 12.08.1993, Zwierzyniec, 4qq, 1ð, 12.08.1993, Górecko Stare 
(Lubelskie prov.) (from C. violaceus); 19, 15.08.1986, Zalesie n. Limanowa (Malopolskie prov.) (C. violaceus); 1q, 15d, 22.06.1994, Sokolowslo n. Walbrzych (C. violaceus), 4q9, 5 đ̊, 16.09.1991, Rudno n. Wolów (C. coriaceus), 299, 2d, 24.09.1990, Leśna Woda n. Olawa (C. violaceus), 1§, 5.09.2004, Międzygórze n. Bystrzyca Klodzka (Dolnoślasskie prov.); $1 \precsim$, 26.05.1988, Przylubie n. Solec Kujawski (C. coriaceus), 1ð, 8.06.1988, Stary Wierzchlas n.

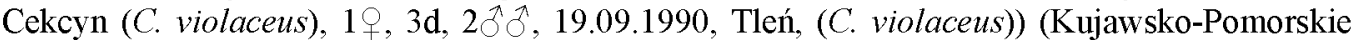
prov.); 18 + $+, 5 \AA \delta, 5.08 .1990$, Szymbark n. Kartuzy, 1ठ, 1d 6.08.1990, Przytuly n. Szymbark (C. violaceus) (Pomorskie prov.); $39+, 3 \hat{\jmath}$, 3d, 15.05.1991, Nowogród Bobrzański (C. violaceus) (Lubuskie prov.); $39+2 \hat{\jmath}, 4 \mathrm{~d}, 18.08 .1990$, Kluczewo n. Czaplinek (C. coriaceus) (Zachodniopomorskie); 4우, 1d, 3.10.1984, Lubsza n. Brzeg (C. violaceus) (Opolskie prov.); 1ㅇ, 3d, 5.08.1996, Kamionek Wielki n. Tolkmicko (C. coriaceus)(Warmińsko-Mazurskie prov.).

Hosts: Carabus coriaceus Linnaeus, 1758, C. violaceus Linnaeus, 1758.

Distribution: Czech Republic, Moldova, Poland, Ukraine (Samšiňák, 1971, Haitlinger, 1988d, Trach, 2006).

Photia Oudemans, 1904

\section{Photia chrysocarabi Cooreman, 1950}

Localities: Taszów n. Duszniki Zdrj (Dolnośląskie prov.), Babia Góra, Zawoja-Policzne (Malopolskie prov.), Widelki n. Bereżk, Ustrzyki Górnei (Podkarpackie prov.) (Haitlinger, $1988 \mathrm{~d})$.

New localities: $6 q q, 2 \AA \hat{\jmath}, 3 \mathrm{~d}, 9.06 .1992$, Jugów n. Nowa Ruda, 25qq, 8ภð, 21.06.1989, Kletno n. Stronie Śląskie, 18 +q, $8 \hat{\delta} \hat{\partial}, 25.05 .1989$, Pasterka n. Kudowa, 12 $q 0,7 \hat{\delta}, 10 \mathrm{~d}$, 21.06.1989, Ostra Góra n. Pasterka (Dolnośląskie prov.), 55q, 38 §̧, 75d, 24.09.1993, Grabarze n. Dzialoszyn (Łódzkie prov.), 17q+, 8§え, 1d, 5.07.1993, Kamesznica Górna (Śląskie prov.); 8qq, 9.07.1989, Brzegi n. Bukowina, $7 q q, 8 \AA \widehat{\partial}, 8.06 .1991$, Lejowa Dolina (Tatry) (Malopolskie prov.);

Hosts: Carabus auroniitens Fabricius, 1792; accidental hosts: C. irregularis Fabricius, 1792; C. scheidleri Panzer, 1799

Distribution: Austria, Belgium, Czech Republic, England, France, Germany, Poland, Slovakia, Ukraine (Cooreman, 1950, Samšiňák, 1971, Haitlinger, 1988d, 1995, Trach, 2006).

\section{Photia hejniana Samšiňák, 1971}

Localities: Ostrzyca n. Proboszczów, Gola Wielka n. Twardogóra, Osola n. Oborniki Śląskie (Dolnośląskie prov.), Rawicz, Klempicz n. Wronki , Podgaje n. Jastrowie (Wielkopolskie prov.), Kamień Śląski (Opolskie prov.), Studzianki Pancerne, Jachranka (Mazowieckie prov.), Bieszczady (Podkarpackie prov.) (Haitlinger, 1988d).

New localities:. 11qq, $9 \hat{\partial} \hat{\partial}, 4 d, 5.07 .1993$, Kamesznica Górna, 5qq, 66.07.1989, Wisła-

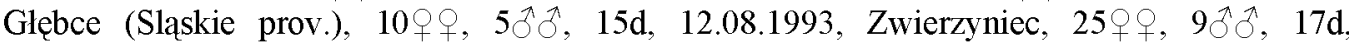
11.08.1993, Górecko Stare (Lubelskie prov,.); 69, 8§̧, 16.07.1993, Zalesie n. Limanowa,

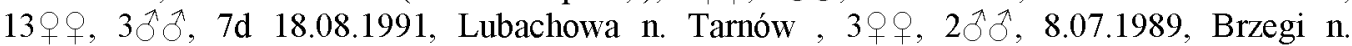
Bukowina, $29 q, 13.07 .1989$, Hańczowa n. Uście Gorlickie, $19 \partial \partial$ f 6.07.1993, Wieprzowiec n. Maków Podhalański (Malopolskie prov.); $22+q, 9 \hat{\jmath}, 2 \mathrm{~d}, 24.09 .1993$, Grabarze n. Dzialoszyn (Lódzkie prov.); 2 $+9,3 \hat{\jmath}$, 1.08.1993, Szczawne n. Sanok, 4+q, 15.08.1991, Bystre n.

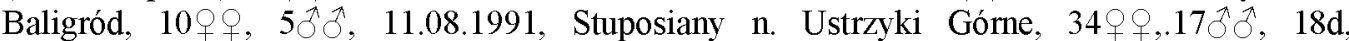
1.08.1993, Wysoczany n. Komańcza, $29+, 22.07 .1993$, Chyrowa n. Dukla, 1 9 , ld, 12.08.1991, Ustrzyki Górne, 24우, 18 đో, 5d, 12.07.1987, Kamionka n. Sędziszów Małopolski, 16우, 6

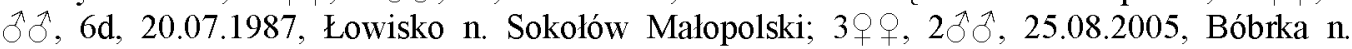


Krosno (Podkarpackie prov.); 1ð, 22.06.1994, Sokolowsko n. Walbrzych, 1q, $3 \delta \AA, 2 \mathrm{~d}$,

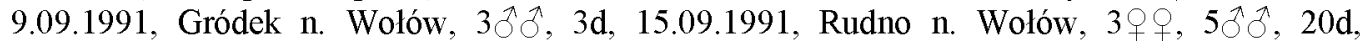

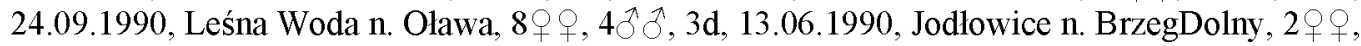
$1 \AA$, 1d, 7.07.1992, Jordanów (Dolnośląskie prov.); 32q9, 18ठð, 7d, 8.06.1988, Stary Wierzchlas n. Tuchola, $119 q, 3 \delta \delta, 7 d, 8.06 .1988$, Stary Wierzchucin n. Cekcyn, $290,2 \delta \delta$, 19.09.1990, Tleń, 17우, 12 đ̊, 3d. 2.08.1990, Ciche n. Brodnica (Kujawsko-Pomorskie prov.);

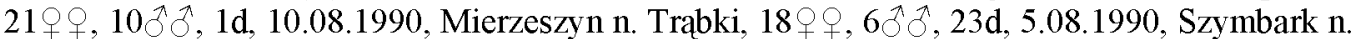
Kartuzy, 5우, 3d, 13.08.1990, Objazda n. Ustka, 6우, 3đđđ, 1d, 6.08.1990, Przytuly n.

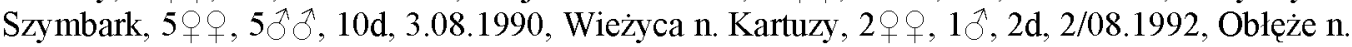
Kępice (Pomorskie prov.), $3+9,3 \hat{\jmath}, 5 \mathrm{~d}, 18.07 .1990$, Burdag n. Jedwabno (WarmińskoMazuskie prov.); 20 우, 11 $\hat{\jmath}, 2 \mathrm{~d}, 15.05 .1991$, Nowogród Bobrzański (Lubuskie prov.); 5 우, $1 \hat{\jmath}, 19.08 .1989$, Parszów n. Skarżysko (Świętokrzyskie prov.).

Host: Carabus violaceus Linnaeus, 1758, accidental host: C. coriaceus.

Distribution: Austria, Croatia, Czech Republic, Moldova, Poland, Slovakia, Ukraine (Samšiňák, 1971, Haitlinger, 1988d, 1995, Trach, 2006).

\section{Photia hermengildae Haitlinger, 1988}

Localities: Huta Szklana (Świętokrzyskie prov.), Babia Góra (Małopolskie prov.), Bieszczady (Podkarpackie prov.) (Haitlinger, 1988c).

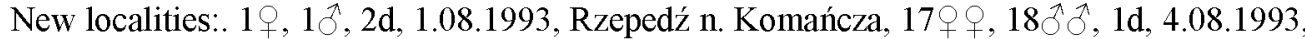
Nowosiólki Dydyńskie n. Przemyśl, 39q, $1 \delta$, 14.07.1999, Kamionka n. Kolbuszowa, 24 9 , $14 \hat{\jmath}$, 12d, 2.08.1991, Gruszowa n. Huwniki, 5ㅇ, 19đ, 22.07.1989, Rymanów Zdrój, 3 우, $3 \hat{\jmath}, 3 d, 1.08 .1993$, Kulaszne n. Komańcza, 11우, $3 \hat{\jmath}, 15.08 .1991$, Bystre n. Baligród

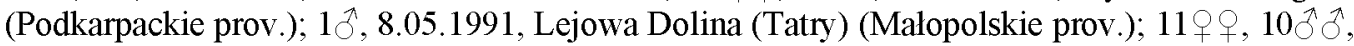
6d, 20.06.1990, Pokrzywna n. Glucholazy (Opolskie prov.); 10 $q+7 \hat{\jmath}, 3 \mathrm{~d}, 12.08 .1883$, Zwierzyniec (Lubelskie prov.)

Host: Carabus linnaei Panzer, 1812.

Distribution: Poland, Ukraine (Haitlinger, 1988c, Trach, 2006).

\section{Photia adolfinae Haitlinger, 1994}

Localities: Narewka n. Hajnówka, Sutno n. Drohiczyn, Białowieża (Podlaskie prov.) Twardogóra, Sulistrowiczki n. Sobótka, Pasterka n. Kudowa Zdrój (Dolnośląskie prov.), Zwierzyniec n. Bilgoraj (Lubelskie prov.), Brzegi n. Bukowina, Zawoja-Markowe, Babia Góra, Spalenica n. Polica (Malopolskie prov.), Mrozy n. Mińsk Mazowiecki (Mazowieckie prov.) (Haitlinger, 1988d 1994).

New localities: 22 + $9,8 d, 9.06 .1992$, Jugów n. Nowa Ruda, $19,2 \widehat{\jmath}, 20.06 .1988$, Kletno n.

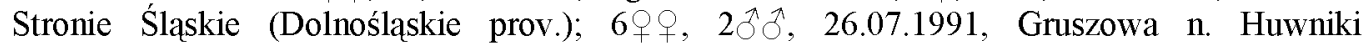
(Podkarpackie prov.), 1, 5.07.1993, Kamesznica Górna (Sląskie prov.)

Hosts: Carabus glabratus, Paykull, 1790,.C. gyllenhali Fisher von Waldheim, 1827.

Distribution: Byelorussia, Poland, Ukraine (Haitlinger, 1994, Khaustov \& Eidelberg, 2001, Trach, 2006).

\section{Photia bardoica Haitlinger, 1988}

Localities: Bardo Śląskie n. Kłodzko, Łagów n. Zgorzelec (Dolnośląskie prov.), Centawa n. Jemielnica (Opolskie prov.), Bieszczady (Podkarpackie prov.) (Haitlinger, 1988c)

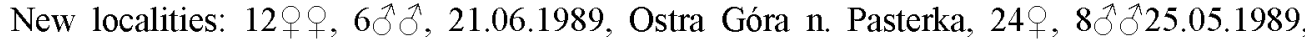
Pasterka n. Kudowa, 2 $9+9,3 \AA \widehat{\jmath}, 3 d, 25.05 .1990$, Karlów n Kudowa (Dolnośląskie prov.); $9 \circ \varnothing, 5 \hat{\jmath}, 25.05 .1988$, Przylubie n. Solec Kujawski (Kujawsko-Pomorskie prov.); 16

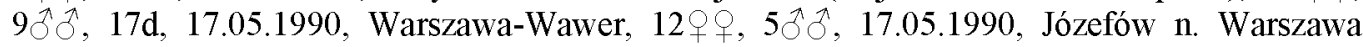


(Mazowieckie prov.); 29q, 1ð, 23.09.1993, Krzeczów n. Dzialoszyn (Łódzkie prov.); 899 ,

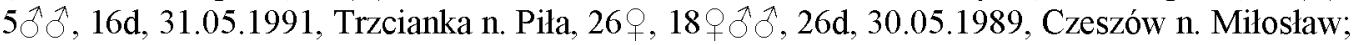

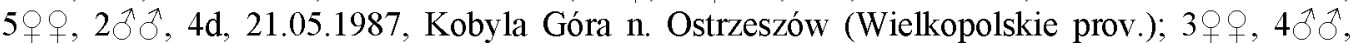
6d19.06.1987, Krzywa Góra n. Pokój (Opolskie prov.); 8우, 9ð̊̉, 2d, 8.07.1992, Lagów (Lubuskie prov.).

Host: Carabus nemoralis O. F. Müller, 1764

Distribution: Austria, Poland (Haitlinger, 1988c, 1995).

\section{SUMMARY}

Among 14 species of canestriniid mites found in Poland, the only 4 species are very common in almost whole country: P. hejniana (known from 14 provinces), $P$. sellnicki (12), P. bourgonei (10) and C. megnini (10). Very rare species are: P. blaptis known only from a single locality in Warmińsko-Mazurskie prov., D. knobi from Malopolskie prov., D. huberti from Podkarpackie prov., P. albini from Pomorskie prov. and Zachodniopomorskie prov. and P. mahunkai known from Dolnoślaskie prov. and Wielkopolskie prov. All these species are associated with rare host species in Poland. P. chrysocarabi and $P$. hermengildae belong to frequently collected species but both these species are restricted only to south and middle parts of the country. The presence of all canestriniid mites is closely dependent of their host territory. The 13 species occurring in Poland are associated with one host. The only $C$. sellnicki is associated with two hosts: $C$. coriaceus and $C$. violaceus. C. megnini was collected from two hosts but C. aurata is accidental host. In other countries for $D$. knobi, P. blaptis, P. chrysocarabi, $C$. negnini, $C$. albinii and $P$. adolfinae also other host species were stated (Khaustov \& Eidelberg 2001, Trach, 2006, Trach \& Khaustov, 2011).

The list of Polish Canestriniidae is not closed. At least species belonging to the genera Amansiella and Paramansia, associated with Chrysolina and Timarcha species can be found in future.

\section{KEY TO SPECIES OF THE GENUS PHOTIA OF THE WORLD (FEMALES)}

1. Setae $\mathrm{c} 1$ at least twice shorter than setae $\mathrm{d} 1$ 2

- Setae cl somewhat shorter than $\mathrm{d} 1$, equal in length or longer than $\mathrm{d} 1$

2. Setae d1 about twice longer than setae c1, host: Carabus scabrinus, C. syriacus

Photia procera (Berlese, 1911);

- Setae d1 at least 3 times longer than setae c1

3. Tops of setae d1 reach beyond posterior margin of idiosoma; hosts: C. impressus, $C$. anatolicus, C. coriaceus Photia procustidis (Berlese, 1881)

- Tops of setae d1 not reach beyond posterior margin of idiosoma; hosts: C. coriaceus,

C. kindermani Photia graeca Cooreman, 1958

4. Setae $\mathrm{c} 1$ and d1 placed almost on the same level ...................................................... 5

- Setae $\mathrm{cl}$ and d1 placed on different level .......................................................... 6

5. The distance between bases of setae $\mathrm{cl}-\mathrm{d} 1$ and $\mathrm{cl}-\mathrm{c} 1$ almost equal; hosts: $C$. coriaceus, $C$. violaceus Photia saetolata (Cooreman, 1959)

- The distance between bases of setae $\mathrm{cl}$ - $\mathrm{cl}$ distinctly greater than distance between bases of setae c1-d1; host: C. violaceus Photia hejniana Samšsiňak, 1971

6. Setae $c 1, c 2, d 1, d 2$, e1, hl very short; their length is subequal (somewhat longer are only d1 and d2); hosts: C. protensus, C. komarovi, C. plasoni, C. schamyli

- These setae (or some of them) distinctly longer with different length

7. Setae $\mathrm{cl}$ and dl short (shorter than $100 \mu \mathrm{m}$ ) 
- Setae $\mathrm{c} 1$ and $\mathrm{d} 1$ long (longer than $100 \mu \mathrm{m}$ )

8. Setae $\mathrm{c} 1<40 \mu \mathrm{m}(30-37), \mathrm{d} 1<45 \mu \mathrm{m}(37-41)$; host: $C$. schoenherri

Photia sibirica Khaustov \& Eidelberg, 2001

- Setae $\mathrm{cl}>40 \mu \mathrm{m}, \mathrm{d} 1>46 \mu \mathrm{m}$

9. Setae c2 $<80 \mu \mathrm{m}(63-75)$; host: C. kolbei ....... Photia pacifica Khaustov \& Eidelberg, 2001

- Setae $\mathrm{c} 2>80 \mu \mathrm{m}$

10. Bases of setae d2 placed posterior to bases of setae $\mathrm{cl}$; setae h1 do not reach posterior margin of idiosoma

- Bases of setae $\mathrm{d} 2$ placed anterior to bases of setae $\mathrm{cl}$; setae $\mathrm{h} 1$ reach posterior margin of idiosoma

11. Setae c2 91-116, d2 136-149; host: C. lopatini

Photia lopatini Khaustov \& Eidelberg, 2001

- Setae c2 280-332, d2 264-296; host: Caraabus sp. ........ Photia melchiori Haitlinger, 1998

12. Setae $\mathrm{cl}>55 \mu \mathrm{m}(58-76)$; ratio IL (length of idiosoma)/cl $<9$; host: C. glabratus

Photia adolfinae Haitlinger, 1994

- Setae cl $<55 \mu \mathrm{m}$, ratio IL/cl > 9; hosts: Carabus sp. .. Photia polymorpha Samšiňák, 1971

13. Setae $\mathrm{cl}$ very long $(>200 \mu \mathrm{m})$, their tops reach far beyond bases of setae. $\mathrm{dl}$; host: Clusitanus Photia lusitanica Samšiňák, 1971

- Tops of setae cl do not reach bases of setae dl, or somewhat exceed these bases ............ 15

14. Length of tarsus IV lesser than $105 \mu \mathrm{m}$, length of posterior seta of anal region ((adl) over $74 \mu \mathrm{m}$; host: $C$. nemoralis Photia bardoica Haitlinger

- Length of tarsus IV over $105 \mu \mathrm{m}$, length of seta ad 1 lesser than $70 \mu \mathrm{m}$ 15

15. Setae vi over $55 \mu \mathrm{m}$ long, distance $\mathrm{d} 1-\mathrm{d} 1$ over $100 \mu \mathrm{m}$, distance el - el over $105 \mu \mathrm{m}$; host: C. auronitens Photia chrysocarabi Cooreman, 1950

- Setae vi less than $55 \mu \mathrm{m}$ long, distance $\mathrm{d} 1$ - dl less than $90 \mu \mathrm{m}$, distance el - el less than $105 \mu \mathrm{m}$; host: C. linnaei Photia hermengildae Haitlinger, 1988

\section{KEY TO SPECIES OF THE GENUS PHOTIA OF THE WORLD (MALES)}

1. Adanal suckers placed side by side 2

- Adanal suckers placed not side by side ....................................................................... 8

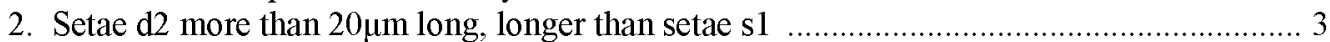

- Setae d2 less than $20 \mu \mathrm{m}$ long, subequal with $\mathrm{cl}$ in length ................................................ 4

3. Dorsum of idiosoma without reticular pattern, caudal capsule present, setae cl reach the bases of setae dl Photia lopatini Kaustov \& Eidelberg, 2001

- Dorsum of idiosoma with well developed pattern, setae $\mathrm{cl}$ far not reach the bases of setae dl Photia procustidis (Berlese, 1881)

4. Bases of setae c1 placed almost the same level as bases of setae $\mathrm{c} 2$

Photia graeca Cooreman, 1958

- Bases of setae $\mathrm{c} 1$ far beyond of bases of setae $\mathrm{c} 2$

5. Genital apparatus reach the anal opening Photia polymorpha Samšiňák, 1971

- Genital apparatus do not reach anal opening

6. Lateral parts of dorsum with well-developed elongate reticule pattern Photia sibirica Khaustov \& Eidelberg, 2001

- Lateral part of dorsum without reticule pattern

Photia pacifica Khaustov \& Eidelberg, 2991

7. Distance between adanal suckers more than 1.5 diameter of adanal suckers 8

- Distance between adanal suckers distinctly lesser

8. Idiosoma oval without ornamentation Photia bilkorum Samšiňàk, 1971 
- Idiosoma distinctly narrow in its posterior part with ornamentation

Photia procera Berlese, 1911

9. Genital apparatus placed far of opening slit, do not reach its anterior margin ................. 10

- Genital apparatus placed near anterior margin of opening slitor reach to it .................... 11

10. Setae ps 2 about twice longer than setae ps3 .............. Photia saetolata (Cooreman, 1950)

- Setae ps2 and ps3 subequal in length .............................. Photia hejniana Samšiňák 1971

11. Setae $\mathrm{cl}$ and $\mathrm{d} 1$ more than $40 \mu \mathrm{m}$ long ......................... Photia lusitanica Samšiňàk, 1971

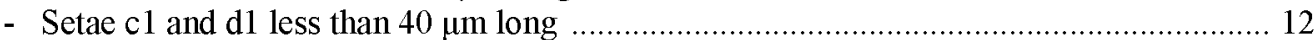

12. TaI less than $58 \mu \mathrm{m}$ long ............................................... Photia bardoica Haitlinger, 1988

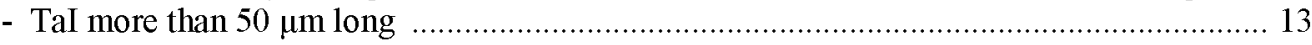

13. Genital apparatus less than $104 \mathrm{~m}$ long, setae $\mathrm{cl}$ and $\mathrm{dl}$ less than $20 \mu \mathrm{m}$ long

Photia adolfinae Haitlinger, 1994

- Genital apparatus more than $106 \mu \mathrm{m}$ long, setae $\mathrm{cl}$ and $\mathrm{dl}$ more than $28 \mu \mathrm{m}$ long ...........14

14. Setae vi less than $50 \mu \mathrm{m}$ long, genital apparatus less than $130 \mu \mathrm{m}$ long

Photia hermengildae Haitlinger, 1988

- Setae vi more than $50 \mu \mathrm{m}$ long, genital apparatus more than $130 \mu \mathrm{m}$ long

Photia chrysocarabi Cooreman, 1950

\section{REFERENCES}

COOREMAN J. 1950. Etude de quelques Canestriniidae (Acari) vivant sur Chrysomelidaeet sur des Carabidae (Insecta: Coleoptera). Bulletin Institut royal des Sciences naturelles de Belgique 26: 1-54.

Griffiths D. A., ATyEo W. T., NORTON R. A. \& LyNCH C. A. 1990. The idiosomal chaetotaxy of astigmatid mites. Journal of Zoology 220: 1-32.

HAITLINGER R. 1988a. Canestrinia dorcicola Berlese, 1881 i Neomyobia chiropteralis (Michael, 1884) (Acari: Canestriniidae, Myobiidae) dwa gatunki roztoczy nowe dla Polski. Przegląd Zoologiczny 32: 41-45.

HAITLINGER R. 1988b. Dicanestrinia knobi Samšiňák, 1971, Coleopterophagus megnini (Berlese, 1881) (Acari, Astigmata, Canestrinitdae) dwa gatunki roztoczy nowe dla fauny Polski. Przegląd Zoologiczny 32: 535-540.

HAItLINGER R. 1988c. Two new species Photia genus Oudemans 1904 (Acari, Astigmata, Canestriniidae) from Poland. Wiadomości Parazytologiczne 34: 319-328.

HAITLINGER R. 1988d. Roztocze (Acari) występujące w Polsce na chrząszczach z rodzaju Carabus Linnaeus, 1758 (Insecta, Coleoptera, Carabidae). Wiadomości Parazytologiczne 34: 329-346.

HAITLINGER R. 1990. The genus Coleopterophagus Berlese, 1882 (Acari, Astigmata, Canestrinitada) with descriptions of seven new species and with key for species determination. Annales Zoologici 43: 327-341.

HAitlinger R.. 1992. The genus Percanestrinia Berlese, 1911 (Acari, Astigmata, Canestriniidae) with descriptions of three new species. Zoologischer Jahrbücher für Systematik 119: 535-542.

HAITLINGER R. 1994. New species of mites (Acari, Astigmata, Canestriniidae) associated with Lucanidae, Carabidae and Scarabaeidae (Insecta, Coleoptera). Wiadomości Parazytologiczne 40: 193-213.

HAITLINGER R. 1995. New mites (Acarina, Astigmata: Canestriniidae; Prostigmata: Erythraeidae, Trombidiidae, Microtrombidiidae) for the fauna of Austria, Germany and Herzegovina with descriptions of four new species. Linzer Biologische Beitrage 27: 259-272.

HAITLINGER R. 2002. Mites (Acarina) associated with Cetoniinae and Triichinae (Insecta; Coleoptera: Scarabaeidae) in Poland, pp. 63-73. In: Ignartowicz S. (ed.), Postępy Polskiej Akarologii. SGGW, Warszawa.

HAitLINGER R. 2004. Mites (Acari) occurring on some Coleoptera (Insecta) in Poland. Polskie Pismo Entomologiczne $73: 3-24$.

KHAUstov A. A. \& EIdelberg M. M. 2001. A review of the mite family Canestriniidse (Acarina: Astigmata) of the eastern Palearctic. Acarina 9: 23-46.

SAMŠIŇÁK K. 1965. Die auf Procrustes (Col. Carab.) lebenden Milben (Acari) und ihre bedeutung fü die Zoogeographie. Mitteilungen aus dem Zoologishen Museum in Berlin 41: 137-155.

SAMŠLŇÁK K. 1971. Die auf Carabus-Arten (Coleoptera, Adephaga) der palearktischen Region lebenden Milben der Unterordnung Acariformes (Acari); ihre Taxonomie und Bedeutuing für die Lösung zoogeograpischer, entwicklungsgeschichtlicher und parasitophyletischer Fragen. Entomologische Abhandlungen Staatliches. Museum Tierkunde in Dresden 38: 145-234.

TRACH V. A. 2006. The host parasite associations of the mites family Canestriniidae (Acari: Astigmata) in Ukraine and Moldova. Vessnik Zoologii 11: 174-180.

Trach V. A. \& Khaustov A. A. 2011. A review of the genus Coleopterophagus Berlese, 1882 (Acari: Astigmata: Canestriniidae) of Ukraine. Acarina 19: 213-230. 
STRESZCZENIE

[Canestriniidae (Acari, Astigmata) Polski, z kluczem do oznaczania roztoczy z rodzaju Photia Oudemans, 1904]

W Polsce stwierdzono obecność 14 gatunków Canestriniidae, reprezentujących 7 rodzajów. Photia hejniana $i$ P. chrysocarabi występują najliczniej. Tylko P. hejniana zostala znaleziona prawie w calej Polsce. Percanestrinia blaptis, Dicanestrinia knobi, D. huberti, Coleopterophagus albini i Pseudocanestrinia mahunkai sa w Polsce bardzo rzadkie. Wszystkie te gatunki są zwiazzane z rzadkimi gatunkami żywicielskimi. Opracowano klucze dla samców i samic do oznaczania wszystkich gatunków z. rodzaju Photia. 